This is an electronic reprint of the original article. This reprint may differ from the original in pagination and typographic detail.

Author(s): Anjam, Immanuel; Mali, Olli; Muzalevsky, Alexey; Neittaanmäki, Pekka; Repin, Sergey

Title: $\quad$ A posteriori error estimates for a Maxwell type problem

Year: $\quad 2009$

Version:

Please cite the original version:

Anjam, I., Mali, O., Muzalevsky, A., Neittaanmäki, P., \& Repin, S. (2009). A posteriori error estimates for a Maxwell type problem. Russian Journal of Numerical Analysis and Mathematical Modelling, 24(5), 395-408.

https://doi.org/10.1515/RJNAMM.2009.025

All material supplied via JYX is protected by copyright and other intellectual property rights, and duplication or sale of all or part of any of the repository collections is not permitted, except that material may be duplicated by you for your research use or educational purposes in electronic or print form. You must obtain permission for any other use. Electronic or print copies may not be offered, whether for sale or otherwise to anyone who is not an authorised user. 


\title{
A posteriori error estimates for a Maxwell type problem
}

\author{
I. ANJAM*, O. MALI*, A. MUZALEVSKY ${ }^{\dagger}$, P. NEITTAANMÄKI*, \\ and S. REPIN
}

\begin{abstract}
In this paper, we discuss a posteriori estimates for the Maxwell type boundary-value problem. The estimates are derived by transformations of integral identities that define the generalized solution and are valid for any conforming approximation of the exact solution. It is proved analytically and confirmed numerically that the estimates indeed provide a computable and guaranteed bound of approximation errors. Also, it is shown that the estimates imply robust error indicators that represent the distribution of local (inter-element) errors measured in terms of different norms.
\end{abstract}

\section{Introduction}

In classical settings the Maxwell problem is defined by $\mathbf{E}, \mathbf{D}$ (electric field and induction), $\mathbf{H}$ and $\mathbf{B}$ (magnetic field and induction) satisfying

$$
\begin{aligned}
& \frac{\partial \mathbf{D}}{\partial t}-\operatorname{curl} \mathbf{H}=-\mathbf{J} \\
& \frac{\partial \mathbf{B}}{\partial t}+\operatorname{curl} \mathbf{E}=0
\end{aligned}
$$

for all $(t, \mathbf{x})$ in $(0, T) \times \Omega$. Here $\Omega$ is a bounded and connected domain in $\mathbb{R}^{d}$ with the Lipschitz boundary $\partial \Omega$, and $\mathbf{J}$ is the applied current. Using the constituent relations

$$
\begin{aligned}
& \mathbf{D}=\varepsilon \mathbf{E} \\
& \mathbf{B}=\mu \mathbf{H}
\end{aligned}
$$

where $\varepsilon(\mathbf{x})>0$ is the dielectric permittivity and $\mu(\mathbf{x})>0$ is the magnetic permeability (both $\mu$ and $\varepsilon$ are assumed to be positive constants or positive bounded

\footnotetext{
* Department of Mathematical Information Technology, University of Jyvaskyla, Fi-40014, Finland

$\dagger$ Applied Mathematics Department, St. Petersburg State Polytechnic University, St. Petersburg 195251, Russia

†St. Petersburg Department of V. A. Steklov Institute of Mathematics, St. Petersburg 191023, Russia
} 
functions), we can rewrite the Maxwell equations in terms of $\mathbf{E}$ and $\mathbf{H}$ only:

$$
\begin{aligned}
& \varepsilon \frac{\partial \mathbf{E}}{\partial t}-\operatorname{curl} \mathbf{H}=-\mathbf{J} \\
& \mu \frac{\partial \mathbf{H}}{\partial t}+\operatorname{curl} \mathbf{E}=0 .
\end{aligned}
$$

These equations must be accompanied by initial conditions and suitable boundary conditions. In this paper, we assume that $\mathbf{E}$ satisfies the so-called PEC (perfect electric conductor) boundary condition

$$
\mathbf{E} \times \mathbf{n}=0 \quad \text { on } \partial \Omega
$$

where $\mathbf{n}$ denotes the unit outward normal to $\partial \Omega$. Usually the time derivatives are replaced by incremental relations. Using the backward-Euler scheme we have

$$
\begin{aligned}
\frac{\varepsilon}{\triangle t}\left(\mathbf{E}^{n}-\mathbf{E}^{n-1}\right)-\operatorname{curl} \mathbf{H}^{n} & =-\mathbf{J} \\
\frac{\mu}{\triangle t}\left(\mathbf{H}^{n}-\mathbf{H}^{n-1}\right)+\operatorname{curl} \mathbf{E}^{n} & =0, \quad n=1, \ldots, N, \quad N=\frac{T}{\triangle t}
\end{aligned}
$$

where $\triangle t$ is the timestep. By eliminating $\mathbf{H}^{n}$ and transferring $\mathbf{E}^{n-1}$ and $\mathbf{H}^{n-1}$ to the right-hand side, we have

$$
\operatorname{curl}\left(\mu^{-1} \operatorname{curl} \mathbf{E}^{n}\right)+\frac{\varepsilon}{(\triangle t)^{2}} \mathbf{E}^{n}=\frac{1}{\triangle t}\left(-\mathbf{J}+\frac{\varepsilon}{\triangle t} \mathbf{E}^{n-1}+\operatorname{curl} \mathbf{H}^{n-1}\right) .
$$

We denote the right-hand side by $\mathbf{f} \in L_{2}\left(\Omega, \mathbb{R}^{d}\right)$, set $\varkappa=\varepsilon(\triangle t)^{-2}$ and arrive at the model problem

$$
\begin{array}{rlrl}
\operatorname{curl}\left(\mu^{-1} \operatorname{curl} \mathbf{E}\right)+\varkappa \mathbf{E} & =\mathbf{f} & & \text { in } \Omega \\
\mathbf{E} \times \mathbf{n}=0 & & \text { on } \partial \Omega
\end{array}
$$

in which the superscript $n$ is omitted.

Below, we study (1.1)-(1.2) in the 2D case, so that the double curl is understood as curl curl, where

$$
\operatorname{curl} \mathbf{w}:=\partial_{1} w_{2}-\partial_{2} w_{1}, \quad \underline{\operatorname{curl}} \varphi:=\left(\begin{array}{c}
\partial_{2} \varphi \\
-\partial_{1} \varphi
\end{array}\right)
$$

We denote by $V(\Omega)$ the space $H(\operatorname{curl} ; \Omega)$. This is a Hilbert space endowed with the norm

$$
\|\mathbf{w}\|_{\text {curl }}=\left(\|\mathbf{w}\|^{2}+\|\operatorname{curl} \mathbf{w}\|^{2}\right)^{1 / 2}
$$


Here $\|\cdot\|$ is the $L_{2}$-norm of scalar- and vector-valued functions. By $V_{0}(\Omega)$ we denote a subspace of $V(\Omega)$ that consists of all the functions from $V$ which satisfy boundary condition (1.2), i.e.,

$$
V_{0}:=\{\mathbf{w} \in V \mid \mathbf{w} \times \mathbf{n}=0 \text { on } \partial \Omega\} .
$$

The generalized solution $\mathbf{E} \in V_{0}$ of (1.1)-(1.2) is then defined by the integral relation

$$
\int_{\Omega}\left(\mu^{-1} \operatorname{curl} \mathbf{E} \operatorname{curl} \mathbf{w}+\varkappa \mathbf{E} \cdot \mathbf{w}\right) \mathrm{d} \mathbf{x}=\int_{\Omega} \mathbf{f} \cdot \mathbf{w} \mathrm{d} \mathbf{x} \quad \forall \mathbf{w} \in V_{0} .
$$

Also, we assume that $\mathbf{f}$ is a divergence-free function, so that

$$
\int_{\Omega} \mathbf{f} \cdot \nabla \phi \mathrm{d} \mathbf{x}=0 \quad \forall \phi \in \stackrel{\circ}{H}^{1}(\Omega)
$$

and

$$
0<\mu_{\ominus} \leqslant \mu(\mathbf{x}) \leqslant \mu_{\oplus}
$$

Our goal is to obtain computable bounds of the difference between $\mathbf{E}$ and any function $\widetilde{\mathbf{E}} \in V_{0}$ measured in terms of the weighted (energy) norm

$$
|[\mathbf{w}]|_{(\gamma, \delta)}:=\int_{\Omega}\left(\gamma|\operatorname{curl} \mathbf{w}|^{2}+\delta|\mathbf{w}|^{2}\right) \mathrm{d} \mathbf{x} .
$$

A posteriori error estimation for the Maxwell's equations is a relatively new field of study. Most of the results that have been earlier obtained are based on the residual approach. In particular, residual type error estimates were studied in $[1,6,7]$ and an equilibrated residual approach was presented in [2]. A posteriori estimates for nonconforming approximations for $H$ (curl)-elliptic partial differential equations were studied in [4]. A Zienkiewicz-Zhu type error estimate equivalent to the residual estimate in [1] was introduced in [5].

A posteriori estimates of the functional type present an efficient approach to the problem (a consequent exposition of the corresponding theory is given in $[10,11]$ ). These estimates do not rely on any properties of the numerical method used to compute approximate solutions. This means that a posteriori estimates of the functional type are valid for any conforming approximation. Another important property of such estimates is that they do not contain mesh-dependent constants.

Functional type estimates for the Maxwell problem were derived in [3, 9, 12]. The equation (1.1) with $\varkappa>0$ and $\varkappa=0$ is considered in [12]. The upper bound for the case $\varkappa>0$ does not contain a gap between the estimate and the true error (the estimate is sharp), but is sensitive with respect to small values of $\varkappa$. For the case $\varkappa=0$ the sharpness of the presented upper bound cannot be proven. The same upper bound for the case $\varkappa>0$ is presented in [3]. In addition, in [3] an upper bound for the case of complex $\varkappa, \mathscr{R}(\varkappa) \geqslant 0$ is presented. The sharpness of this upper bound cannot be proven. In [9] a sharp lower bound for $\varkappa>0$ and two new upper bounds are presented. The first new upper bound is for $\varkappa \geqslant 0$ and it is insensitive with 
respect to small values of $\varkappa$. However, this estimate is sensitive with respect to large values of $\varkappa$ and the sharpness of this estimate cannot be proven. The second upper bound is derived in a more sophisticated way and provides a more general upper bound. Also, it behaves well with respect to small and large values of $\varkappa$.

In this paper, we derive functional a posteriori estimates for a model 2D problem that can be viewed as a simplified version of the Maxwell problem. As in [9], the derivation of estimates is based upon transformations of the corresponding integral identity. We prove that the estimates provide guaranteed and computable error bounds for the difference $\mathbf{E}-\widetilde{\mathbf{E}}$, where $\widetilde{\mathbf{E}} \in V_{0}$ is an approximation to the exact solution $\mathbf{E}$. In the last section, these theoretical results are confirmed by numerical experiments.

\section{Error estimates}

\subsection{Upper bound of the error}

First, we present some auxiliary results that are further used in the derivation of the upper bound.

By the Helmholtz decomposition of a vector-valued function, we represent the exact solution $\mathbf{E}$ as follows:

$$
\mathbf{E}=\mathbf{E}_{0}+\nabla \psi
$$

where $\mathbf{E}_{0}$ is a solenoidal vector-valued function and $\psi \in \stackrel{\circ}{H}^{1}(\Omega)$. Since curl $\nabla \psi=0$, we rewrite (1.3) as follows:

$$
\int_{\Omega} \mu^{-1} \operatorname{curl} \mathbf{E}_{0} \operatorname{curl} \mathbf{w}+\varkappa\left(\mathbf{E}_{0}+\nabla \psi\right) \cdot \mathbf{w} \mathrm{d} \mathbf{x}=\int_{\Omega} \mathbf{f} \cdot \mathbf{w} \mathrm{d} \mathbf{x} .
$$

Next, we make the same decomposition for the trial function and set $\mathbf{w}=\mathbf{w}_{0}+\nabla \phi$. Since

$$
\int_{\Omega} \mathbf{f} \cdot \nabla \phi \mathrm{d} \mathbf{x}=\int_{\Omega} \mathbf{E}_{0} \cdot \nabla \phi \mathrm{d} \mathbf{x}=\int_{\Omega} \mathbf{w}_{0} \cdot \nabla \psi \mathrm{d} \mathbf{x}=0
$$

we observe that

$$
\int_{\Omega}\left(\mu^{-1} \operatorname{curl} \mathbf{E}_{0} \operatorname{curl} \mathbf{w}_{0}+\varkappa \mathbf{E}_{0} \cdot \mathbf{w}_{0}+\varkappa \nabla \psi \cdot \nabla \phi\right) \mathrm{d} \mathbf{x}=\int_{\Omega} \mathbf{f} \cdot \mathbf{w}_{0} \mathrm{~d} \mathbf{x} .
$$

By setting $\mathbf{w}_{0}=0$ and $\phi=\psi$, we find that $\|\nabla \psi\|=0$. Hence, $\mathbf{E}$ is a divergence-free function.

Note that $\phi$ satisfies the relation

$$
\int_{\Omega} \nabla \phi \cdot \nabla \xi \mathrm{d} \mathbf{x}=\int_{\Omega} \mathbf{w} \cdot \nabla \xi \mathrm{d} \mathbf{x}=-\int_{\Omega}(\operatorname{div} \mathbf{w}) \xi \mathrm{d} \mathbf{x} \quad \forall \xi \in \stackrel{\circ}{H^{1}}(\Omega)
$$

which implies the estimate

$$
\|\nabla \phi\| \leqslant C_{\Omega}\|\operatorname{div} \mathbf{w}\|
$$


where $C_{\Omega}$ is the constant in the Friedrichs inequality for the domain $\Omega$. For solenoidal fields we also have the estimate (see, e.g., $[7,13]$ )

$$
\left\|\mathbf{w}_{0}\right\| \leqslant C_{\Omega}\left\|\operatorname{curl} \mathbf{w}_{0}\right\|=C_{\Omega}\|\operatorname{curl} \mathbf{w}\| .
$$

Green's formula in a 2D setting states that for any $y \in H^{1}(\Omega)$ and any $\mathbf{w} \in$ $H(\operatorname{curl} ; \Omega)$

$$
\int_{\Omega} y \operatorname{curl} \mathbf{w} \mathrm{d} \mathbf{x}=\int_{\Omega} \underline{\operatorname{curl}} y \cdot \mathbf{w} \mathrm{d} \mathbf{x}+\int_{\partial \Omega} y(\mathbf{w} \times \mathbf{n}) \mathrm{d} s
$$

so we find that

$$
\int_{\Omega}(\underline{\operatorname{curl}} y \cdot \mathbf{w}-y \operatorname{curl} \mathbf{w}) \mathrm{d} \mathbf{x}=0 \quad \forall \mathbf{w} \in V_{0}
$$

Proposition 2.1. Let $\widetilde{\mathbf{E}} \in V_{0} \cap H(\operatorname{div} ; \Omega)$ be an approximation of $\mathbf{E}$. For any $y \in H^{1}(\Omega)$ the following estimate holds:

$$
|[\mathbf{E}-\widetilde{\mathbf{E}}]|_{\gamma, \delta}^{2} \leqslant \mathscr{M}_{\oplus}^{2}\left(\lambda, \alpha_{1}, \alpha_{2}, \widetilde{\mathbf{E}}, y\right)
$$

with

$$
\mathscr{M}_{\oplus}^{2}\left(\lambda, \alpha_{1}, \alpha_{2}, \widetilde{\mathbf{E}}, y\right):=R_{1}(\lambda, \widetilde{\mathbf{E}}, y)+\frac{\alpha_{1}}{4} R_{2}^{2}(\lambda, \widetilde{\mathbf{E}}, y)+\frac{\alpha_{2}}{4} R_{3}^{2}(\lambda, \widetilde{\mathbf{E}}, y)
$$

where $\alpha_{1}$ and $\alpha_{2}$ are arbitrary numbers in $[1,+\infty)$ and $\varkappa$ is a positive constant,

$$
\begin{gathered}
\gamma=\left(1-\frac{1}{\alpha_{1}}\right) \mu^{-1}, \quad \delta=\left(1-\frac{1}{\alpha_{2}}\right) \varkappa \\
\lambda \in I_{[0,1]}:=\left\{\lambda \in L^{\infty}(\Omega) \mid \lambda(\mathbf{x}) \in[0,1] \text { for a.e. } \mathbf{x} \in \Omega\right\}
\end{gathered}
$$

and $R_{i}, i=1,2,3$, are defined by (2.9)-(2.11).

Proof. From (1.3) it follows that

$$
\begin{aligned}
& \int_{\Omega}\left(\mu^{-1} \operatorname{curl}(\mathbf{E}-\widetilde{\mathbf{E}}) \operatorname{curl} \mathbf{w}+\varkappa(\mathbf{E}-\widetilde{\mathbf{E}}) \cdot \mathbf{w}\right) \mathrm{d} \mathbf{x} \\
&=\int_{\Omega}\left(\mathbf{f} \cdot \mathbf{w}-\mu^{-1} \operatorname{curl} \widetilde{\mathbf{E}} \operatorname{curl} \mathbf{w}-\varkappa \widetilde{\mathbf{E}} \cdot \mathbf{w}\right) \mathrm{d} \mathbf{x} .
\end{aligned}
$$

By (2.3) and (2.5) we obtain

$$
\begin{aligned}
\int_{\Omega}\left(\mu^{-1} \operatorname{curl}(\mathbf{E}-\widetilde{\mathbf{E}}) \operatorname{curl} \mathbf{w}+\right. & \varkappa(\mathbf{E}-\widetilde{\mathbf{E}}) \cdot \mathbf{w}) \mathrm{d} \mathbf{x} \\
& =\int_{\Omega} \mathbf{r}(\widetilde{\mathbf{E}}, y) \cdot \mathbf{w} \mathrm{d} \mathbf{x}+\int_{\Omega} d(\widetilde{\mathbf{E}}, y) \operatorname{curl} \mathbf{w} \mathrm{d} \mathbf{x}
\end{aligned}
$$


where

$$
\begin{aligned}
\mathbf{r}(\widetilde{\mathbf{E}}, y) & :=\mathbf{f}-\underline{\operatorname{curl} y-\varkappa \widetilde{\mathbf{E}}} \\
d(\widetilde{\mathbf{E}}, y) & :=y-\mu^{-1} \operatorname{curl} \widetilde{\mathbf{E}} .
\end{aligned}
$$

With the help of $\lambda$ we decompose integral identity (2.6) as follows:

$$
\begin{aligned}
& \int_{\Omega}\left(\mu^{-1} \operatorname{curl}(\mathbf{E}-\widetilde{\mathbf{E}}) \operatorname{curl} \mathbf{w}+\varkappa(\mathbf{E}-\widetilde{\mathbf{E}}) \cdot \mathbf{w}\right) \mathrm{d} \mathbf{x} \\
& =\int_{\Omega} \lambda \mathbf{r}(\widetilde{\mathbf{E}}, y) \cdot \mathbf{w} \mathrm{d} \mathbf{x}+\int_{\Omega}(1-\lambda) \mathbf{r}(\widetilde{\mathbf{E}}, y) \cdot \mathbf{w} \mathrm{d} \mathbf{x}+\int_{\Omega} d(\widetilde{\mathbf{E}}, y) \operatorname{curl} \mathbf{w} \mathrm{d} \mathbf{x}
\end{aligned}
$$

where $\lambda \in I_{[0,1]}$. Since

$$
\int_{\Omega} \lambda \mathbf{r}(\widetilde{\mathbf{E}}, y) \cdot(\mathbf{E}-\widetilde{\mathbf{E}}) \mathrm{d} \mathbf{x} \leqslant\left\|\frac{\lambda}{\varkappa^{1 / 2}} \mathbf{r}(\widetilde{\mathbf{E}}, y)\right\|\left\|\varkappa^{1 / 2}(\mathbf{E}-\widetilde{\mathbf{E}})\right\|
$$

and by inequalities (2.1) and (2.2)

$$
\begin{aligned}
& \int_{\Omega}(1-\lambda) \mathbf{r}(\widetilde{\mathbf{E}}, y) \cdot(\mathbf{E}-\widetilde{\mathbf{E}}) \mathrm{d} \mathbf{x} \\
& \quad \leqslant\|(1-\lambda) \mathbf{r}(\widetilde{\mathbf{E}}, y)\|\left(C_{\Omega}\|\operatorname{div} \widetilde{\mathbf{E}}\|+C_{\Omega} \mu_{\oplus}^{1 / 2}\left\|\mu^{-1 / 2} \operatorname{curl}(\mathbf{E}-\widetilde{\mathbf{E}})\right\|\right) .
\end{aligned}
$$

By setting $\mathbf{w}=\mathbf{E}-\widetilde{\mathbf{E}}$ equation (2.7) becomes

$$
\begin{aligned}
\int_{\Omega}\left(\mu^{-1}|\operatorname{curl}(\mathbf{E}-\widetilde{\mathbf{E}})|^{2}\right. & \left.+\varkappa|\mathbf{E}-\widetilde{\mathbf{E}}|^{2}\right) \mathrm{d} \mathbf{x} \\
& \leqslant R_{1}+R_{2}\left\|\mu^{-1 / 2} \operatorname{curl}(\mathbf{E}-\widetilde{\mathbf{E}})\right\|+R_{3}\left\|\varkappa^{1 / 2}(\mathbf{E}-\widetilde{\mathbf{E}})\right\|
\end{aligned}
$$

where

$$
\begin{aligned}
& R_{1}(\lambda, \widetilde{\mathbf{E}}, y)=C_{\Omega}\|(1-\lambda) \mathbf{r}(\widetilde{\mathbf{E}}, y)\|\|\operatorname{div} \widetilde{\mathbf{E}}\| \\
& R_{2}(\lambda, \widetilde{\mathbf{E}}, y)=C_{\Omega} \mu_{\oplus}^{1 / 2}\|(1-\lambda) \mathbf{r}(\widetilde{\mathbf{E}}, y)\|+\left\|\mu^{1 / 2} d(\widetilde{\mathbf{E}}, y)\right\| \\
& R_{3}(\lambda, \widetilde{\mathbf{E}}, y)=\left\|\frac{\lambda}{\varkappa^{1 / 2}} \mathbf{r}(\widetilde{\mathbf{E}}, y)\right\| .
\end{aligned}
$$

By applying Young's inequality to the right-hand side of (2.8), we obtain

$$
\begin{aligned}
\int_{\Omega}\left(1-\frac{1}{\alpha_{1}}\right) \mu^{-1}|\operatorname{curl}(\mathbf{E}-\widetilde{\mathbf{E}})|^{2} \mathrm{~d} \mathbf{x}+\int_{\Omega} & \left(1-\frac{1}{\alpha_{2}}\right) \varkappa|\mathbf{E}-\widetilde{\mathbf{E}}|^{2} \mathrm{~d} \mathbf{x} \\
& \leqslant R_{1}+\frac{\alpha_{1}}{4} R_{2}^{2}+\frac{\alpha_{2}}{4} R_{3}^{2}
\end{aligned}
$$

which implies (2.4). 
Remark 2.1. A form of $\lambda$ which is optimal (from the theoretical point of view) is obtained in [9], where similar estimates are considered for a 3D problem.

Corollary 2.1. If $\alpha_{1}=\alpha_{2}=2$ then (2.4) comes in the form

$$
|[\mathbf{E}-\widetilde{\mathbf{E}}]|_{\left(\mu^{-1}, \varkappa\right)}^{2} \leqslant \mathscr{M}_{\oplus}^{(\lambda)}
$$

where

$$
\mathscr{M}_{\oplus}^{(\lambda)}:=\mathscr{M}_{\oplus}^{2}(\lambda, \widetilde{\mathbf{E}}, y)=2 R_{1}(\lambda, \widetilde{\mathbf{E}}, y)+R_{2}^{2}(\lambda, \widetilde{\mathbf{E}}, y)+R_{3}^{2}(\lambda, \widetilde{\mathbf{E}}, y)
$$

and this estimate is sharp.

Proof. It holds that

$$
\inf _{\substack{\lambda \in I_{[0,1]} \\ y \in H^{1}(\Omega)}} \mathscr{M}_{\oplus}^{(\lambda)}(\widetilde{\mathbf{E}}, y) \leqslant \inf _{\substack{y \in H^{1}(\Omega)\\}} \mathscr{M}_{\oplus}^{(1)}(\widetilde{\mathbf{E}}, y) \leqslant \mathscr{M}_{\oplus}^{(1)}(\widetilde{\mathbf{E}}, p)
$$

where $p=\mu^{-1}$ curl E. We have

$$
\mathscr{M}_{\oplus}^{(1)}(\widetilde{\mathbf{E}}, p)=\left\|\mu^{-1 / 2} \operatorname{curl}(\mathbf{E}-\widetilde{\mathbf{E}})\right\|^{2}+\left\|\varkappa^{1 / 2}(\mathbf{E}-\widetilde{\mathbf{E}})\right\|^{2}=|[\mathbf{E}-\widetilde{\mathbf{E}}]|_{\left(\mu^{-1}, \varkappa\right)}^{2} .
$$

It means that the estimate is sharp.

Remark 2.2. By setting $\lambda=1$ and $\lambda=0$ we arrive at two particular forms of the error bound, which we call $\mathscr{M}_{\oplus}^{(1)}$ and $\mathscr{M}_{\oplus}^{(0)}$ respectively. They are as follows:

$$
\mathscr{M}_{\oplus}^{(1)}=\left\|\varkappa^{-1 / 2} \mathbf{r}(\widetilde{\mathbf{E}}, y)\right\|^{2}+\left\|\mu^{1 / 2} d(\widetilde{\mathbf{E}}, y)\right\|^{2}
$$

and

$$
\mathscr{M}_{\oplus}^{(0)}=2 C_{\Omega}\|\mathbf{r}(\widetilde{\mathbf{E}}, y)\|\|\operatorname{div} \widetilde{\mathbf{E}}\|+\left(C_{\Omega} \mu_{\oplus}^{1 / 2}\|\mathbf{r}(\widetilde{\mathbf{E}}, y)\|+\left\|\mu^{1 / 2} d(\widetilde{\mathbf{E}}, y)\right\|\right)^{2} .
$$

It should be noted that $\mathscr{M}_{\oplus}^{(0)}$ is well adapted to the case, in which $\varkappa$ is small (or even zero) and may lead to a considerable overestimation if $\varkappa$ is large. Conversely, $\mathscr{M}_{\oplus}^{(1)}$ is sensitive with respect to small $\varkappa$ and is well adapted to large values of this parameter. The combined majorant $\mathscr{M}_{\oplus}^{(\lambda)}$ is applicable to both cases. This property is due to the presence of the function $\lambda$, which allows us to compensate small values of $\varkappa$. 


\subsection{Lower bound of the error}

Proposition 2.2. Assume that $\varkappa>0$ and $\widetilde{\mathbf{E}} \in V_{0}$ is an approximation of $\mathbf{E}$. For any $\mathbf{w} \in V_{0}$ the following estimate holds:

$$
|[\mathbf{E}-\widetilde{\mathbf{E}}]|_{\left(\mu^{-1}, \varkappa\right)}^{2} \geqslant \mathscr{M}_{\ominus}^{2}(\widetilde{\mathbf{E}}, \mathbf{w})
$$

where

$$
\begin{aligned}
\mathscr{M}_{\ominus}^{2}(\widetilde{\mathbf{E}}, \mathbf{w}):=\int_{\Omega}\left(2 \mathbf{f} \cdot \mathbf{w}-\mu^{-1}|\operatorname{curl} \mathbf{w}|^{2}\right. & \\
& \left.-\varkappa|\mathbf{w}|^{2}-2 \mu^{-1} \operatorname{curl} \widetilde{\mathbf{E}} \operatorname{curl} \mathbf{w}-2 \varkappa \widetilde{\mathbf{E}} \cdot \mathbf{w}\right) \mathrm{d} \mathbf{x} .
\end{aligned}
$$

Proof. First, we note that

$$
\begin{aligned}
\sup _{w \in V_{0}} & \int_{\Omega}\left(\mu^{-1} \operatorname{curl}(\mathbf{E}-\widetilde{\mathbf{E}}) \operatorname{curl} \mathbf{w}+\varkappa \mathbf{w} \cdot(\mathbf{E}-\widetilde{\mathbf{E}})\right. \\
& \left.-\frac{1}{2}\left(\mu^{-1} \operatorname{curl} \mathbf{w} \operatorname{curl} \mathbf{w}+\varkappa \mathbf{w} \cdot \mathbf{w}\right)\right) \mathrm{d} \mathbf{x} \\
\leqslant & \sup _{\substack{\tau \in H^{1}(\Omega, \mathbb{R}) \\
\mathbf{w} \in L_{2}\left(\Omega, \mathbb{R}^{2}\right)}} \int_{\Omega}\left(\mu^{-1} \operatorname{curl}(\mathbf{E}-\widetilde{\mathbf{E}}) \tau-\frac{1}{2} \mu^{-1} \tau \tau\right. \\
& \left.+\varkappa \mathbf{w} \cdot(\mathbf{E}-\widetilde{\mathbf{E}})-\frac{1}{2} \varkappa \mathbf{w} \cdot \mathbf{w}\right) \mathrm{d} \mathbf{x}=\frac{1}{2}|[\mathbf{E}-\widetilde{\mathbf{E}}]|_{\left(\mu^{-1}, \varkappa\right)}^{2} .
\end{aligned}
$$

On the other hand,

$$
\begin{aligned}
\sup _{w \in V_{0}} & \int_{\Omega}\left(\mu^{-1} \operatorname{curl}(\mathbf{E}-\widetilde{\mathbf{E}}) \operatorname{curl} \mathbf{w}+\varkappa \mathbf{w} \cdot(\mathbf{E}-\widetilde{\mathbf{E}})\right. \\
& \left.-\frac{1}{2}\left(\mu^{-1} \operatorname{curl} \mathbf{w} \operatorname{curl} \mathbf{w}+\varkappa \mathbf{w} \cdot \mathbf{w}\right)\right) \mathrm{d} \mathbf{x} \\
\geqslant & \int_{\Omega}\left(\mu^{-1} \operatorname{curl}(\mathbf{E}-\widetilde{\mathbf{E}}) \operatorname{curl}(\mathbf{E}-\widetilde{\mathbf{E}})+\varkappa(\mathbf{E}-\widetilde{\mathbf{E}}) \cdot(\mathbf{E}-\widetilde{\mathbf{E}})\right. \\
& \left.-\frac{1}{2}\left(\mu^{-1}|\operatorname{curl}(\mathbf{E}-\widetilde{\mathbf{E}})|^{2}+\varkappa|\mathbf{E}-\widetilde{\mathbf{E}}|^{2}\right)\right) \mathrm{d} \mathbf{x}=\frac{1}{2}|[\mathbf{E}-\widetilde{\mathbf{E}}]|_{\left(\mu^{-1}, \varkappa\right)}^{2} .
\end{aligned}
$$

Thus, we conclude that

$$
\begin{aligned}
\frac{1}{2}|[\mathbf{E}-\widetilde{\mathbf{E}}]|_{\left(\mu^{-1}, \varkappa\right)}^{2}= & \sup _{\mathbf{w} \in V_{0}} \int_{\Omega}\left(\mu^{-1} \operatorname{curl}(\mathbf{E}-\widetilde{\mathbf{E}}) \operatorname{curl} \mathbf{w}\right. \\
& \left.+\varkappa \mathbf{w} \cdot(\mathbf{E}-\widetilde{\mathbf{E}})-\frac{1}{2}\left(\mu^{-1} \operatorname{curl} \mathbf{w} \operatorname{curl} \mathbf{w}+\varkappa \mathbf{w} \cdot \mathbf{w}\right)\right) \mathrm{d} \mathbf{x} .
\end{aligned}
$$

Using equation (1.3), we obtain (2.16). 
Corollary 2.2. The sharpest bound is given by the quantity

$$
\mathscr{M}_{\ominus}^{2}(\widetilde{\mathbf{E}})=\sup _{\mathbf{w} \in V_{0}} \mathscr{M}_{\ominus}^{2}(\widetilde{\mathbf{E}}, \mathbf{w}) .
$$

By setting $\mathbf{w}=\mathbf{E}-\widetilde{\mathbf{E}}$, we find that

$$
\mathscr{M}_{\ominus}^{2}(\widetilde{\mathbf{E}})=|[\mathbf{E}-\widetilde{\mathbf{E}}]|_{\left(\mu^{-1}, \varkappa\right)}^{2}
$$

so the lower bound is sharp.

\section{Numerical results}

Estimates derived in the previous section have been verified in a series of numerical tests, which are discussed in this section. Approximations for the model problem were calculated with lowest-order Nédélec's elements of the first type (e.g., see $[7,8])$. It should be noted that in the derivation of the upper bound we used the Helmholtz decomposition for the numerical approximation of the exact solution. Because of this, we are assuming that the numerical approximation belongs not only to $H$ (curl) but also to $H$ (div). With the lowest-order Nédélec's elements the normal component is not continuous across the element edges, so the divergence of approximate solutions is not square summable. To overcome this problem we chose to force the normal continuity by post-processing the numerical solution. Alternatively, one could use the nodal Courant elements to obtain approximate solutions, which belong to $H^{1} \times H^{1}$. This problem does not arise with the upper bound $\mathscr{M}_{\oplus}^{(1)}$, because it can be derived separately without using Helmholtz decomposition (see $[3,9,12])$. Also the lower bound does not require the square summability of the divergence of the numerical approximation.

The free parameter $y$ was obtained by globally minimizing the upper bounds with respect to $y$. Global minimization results in a finite element problem for $y$, which can be solved with standard nodal finite elements. Increasing the order of elements or using a more refined mesh than the mesh on which the approximate solution was computed results in better values for the upper bounds.

The performance of the upper bounds is measured by the so-called efficiency index

$$
I_{\text {eff }}=\left(\frac{\mathscr{M}_{\oplus}^{(\lambda)}}{|[\mathbf{E}-\widetilde{\mathbf{E}}]|_{\left(\mu^{-1}, \varkappa\right)}^{2}}\right)^{1 / 2} .
$$

To get sensible values for the lower bound, the free parameter $\mathbf{w}$ should be a better approximate solution to the problem than the original approximate solution $\mathbf{v}$. A better solution can be computed by simply refining the mesh and computing a new solution on this mesh. The finer the mesh, the better values for the lower bound we get. 
Table 1.

Problem (3.3): Efficiency index values for different values of $\varkappa$.

\begin{tabular}{lcccccc}
\hline & \multicolumn{3}{c}{ linear $y$} & \multicolumn{3}{c}{ quadratic $y$} \\
\hline$\varkappa$ & $\mathscr{M}_{\oplus}^{(1)}$ & $\mathscr{M}_{\oplus}^{(0)}$ & $\mathscr{M}_{\oplus}^{(\lambda)}$ & $\mathscr{M}_{\oplus}^{(1)}$ & $\mathscr{M}_{\oplus}^{(0)}$ & $\mathscr{M}_{\oplus}^{(\lambda)}$ \\
\hline $10^{-3}$ & 103.79 & 1.98 & 1.98 & 6.35 & 1.07 & 1.07 \\
$10^{-1}$ & 10.42 & 1.98 & 1.98 & 1.18 & 1.07 & 1.06 \\
$10^{0}$ & 3.42 & 1.98 & 1.91 & 1.02 & 1.08 & 1.02 \\
$10^{1}$ & 1.42 & 1.96 & 1.42 & 1.00 & 1.18 & 1.00 \\
$10^{3}$ & 1.00 & 7.14 & 1.00 & 1.00 & 7.05 & 1.00 \\
\hline
\end{tabular}

Table 2.

Problem (3.3) with $\varkappa=10^{-3}$ : The sharpness of the upper bound $\mathscr{M}_{\oplus}^{(1)}$ and the lower bound $\mathscr{M}_{\ominus}$.

\begin{tabular}{lcccccc}
\hline & & \multicolumn{3}{c}{ linear $y$} & \multicolumn{2}{c}{ quadratic $y$} \\
\hline \# elem & $|[\mathbf{E}-\widetilde{\mathbf{E}}]|^{2}$ & $\mathscr{M}_{\ominus}^{2}$ & $\mathscr{M}_{\oplus}^{(1)}$ & $I_{\text {eff }}$ & $\mathscr{M}_{\oplus}^{(1)}$ & $I_{\text {eff }}$ \\
\hline 82 & 0.11908 & & 1897.90 & 126.25 & 7.04419 & 7.69 \\
328 & 0.11908 & 0.08914 & 486.837 & 63.94 & 0.55972 & 2.17 \\
1312 & 0.11908 & 0.11158 & 123.000 & 32.14 & 0.14689 & 1.11 \\
5248 & 0.11908 & 0.11721 & 30.9403 & 16.12 & 0.12083 & 1.01 \\
\hline
\end{tabular}

We are also interested in indicating the error distribution in different norms. The upper bound $\mathscr{M}_{\oplus}^{(1)}$ is the most suitable for this purpose, because it does not contain any constants. Using the two terms in $\mathscr{M}_{\oplus}^{(1)}$ separately we define the following error indicators

$$
\begin{aligned}
& I_{r}(\widetilde{\mathbf{E}}, y)=\left\|\varkappa^{-1 / 2} \mathbf{r}(\widetilde{\mathbf{E}}, y)\right\| \\
& I_{d}(\widetilde{\mathbf{E}}, y)=\left\|\mu^{1 / 2} d(\widetilde{\mathbf{E}}, y)\right\| .
\end{aligned}
$$

By setting $y=\mu^{-1}$ curl $\mathbf{E}$ we see that if the free parameter $y$ is chosen properly, indicator (3.1) should give a good error distribution for the weighed $L_{2}$-norm of the error

$$
\left\|\varkappa^{1 / 2}(\mathbf{E}-\widetilde{\mathbf{E}})\right\| .
$$

Respectively, indicator (3.2) should give a good error distribution for the weighed $H$ (curl)-seminorm of the error

$$
\left\|\mu^{-1 / 2} \operatorname{curl}(\mathbf{E}-\widetilde{\mathbf{E}})\right\|
$$

For indicators (3.1) and (3.2) we also used a gradient averaging technique to compute $y$. It works as follows: for each node we calculate the approximate solution's curl values on the surrounding elements and weigh them by the sizes of respective elements. Then average the values to obtain a value for the node. 
Table 3.

Problem (3.4): Efficiency index values with different mesh-sizes.

\begin{tabular}{lccc}
\hline \# elems & $\mathscr{M}_{\oplus}^{(1)}$ & $\mathscr{M}_{\oplus}^{(0)}$ & $\mathscr{M}_{\oplus}^{(\lambda)}$ \\
\hline 72 & 1.00 & 1.05 & 1.00 \\
246 & 1.00 & 1.04 & 1.00 \\
980 & 1.00 & 1.02 & 1.00 \\
\hline
\end{tabular}

For the first test example we take

$$
\Omega=[0,1]^{2}, \quad \mu \equiv 1, \quad \varkappa>0, \quad \mathbf{f}=\left(\pi^{2}+\varkappa\right)\left(\begin{array}{c}
\sin \left(\pi x_{2}\right) \\
\sin \left(\pi x_{1}\right)
\end{array}\right) .
$$

For this problem we know the exact solution

$$
\mathbf{u}=\left(\begin{array}{l}
\sin \left(\pi x_{2}\right) \\
\sin \left(\pi x_{1}\right)
\end{array}\right)
$$

which is the same for all $\varkappa>0$. Table 1 shows the behaviour of the error majorants $\mathscr{M}_{\oplus}^{(1)}, \mathscr{M}_{\oplus}^{(0)}$, and $\mathscr{M}_{\oplus}^{(\lambda)}$ for different $\varkappa$. For each $\varkappa$ the approximate solution is calculated on a mesh with 82 elements and post-processed so that the divergence of the approximate solution becomes square summable. In the left-hand part of the table, the results correspond to the case in which $y$ is computed by minimizing of majorants on the same mesh as for the approximate solution, using piecewise affine continuous approximation. The right-hand part exposes the results obtained by piece-wise quadratic approximations. It is not surprising that the efficiency indexes in the quadratic case are lower. The number of the degrees of freedom for quadratic approximation of $y$ is roughly 4 times more than for the linear case. Another observation, which follows from Table 1 is that the majorants $\mathscr{M}_{\oplus}^{(1)}$ and $\mathscr{M}_{\oplus}^{(0)}$ may essentially overestimate the error, while $\mathscr{M}_{\oplus}^{(\lambda)}$ keeps small values of the efficiency index for all $\varkappa$. The dependence of upper bounds with respect to $\varkappa$ can also be seen in Fig. 1. The left picture corresponds to the linear approximation of $y$ and the right picture corresponds to the quadratic approximation of $y$. From these results we also see that $\mathscr{M}_{\oplus}^{(1)}$ significantly benefits from using quadratic elements to approximate $y$.

Even though $\mathscr{M}_{\oplus}^{(1)}$ seriously overestimates the error with small values of $\varkappa$, the theory says that it is sharp. In principle, with $\mathscr{M}_{\oplus}^{(1)}$ one should be able to get as low efficiency index values as with $\mathscr{M}_{\oplus}^{(\lambda)}$. To verify this theory, we took the case $\varkappa=10^{-3}$ and calculated the numerical approximation in a mesh with 82 elements. For this test we did not post-process the numerical approximation, because this majorant does not require that the approximate solution belongs to $H$ (div). To test the sharpness of this majorant, we calculated the free parameter $y$ on subsequently refined meshes. The results in Table 2 agree with the theory. The convergence of the 

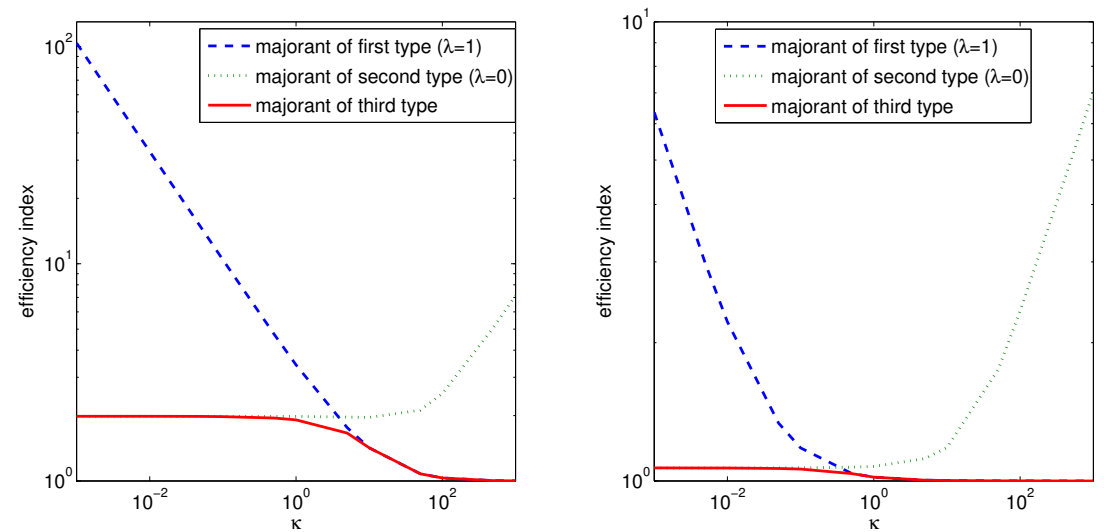

Figure 1. Problem (3.3): Efficiency indexes of the majorants $\mathscr{M}_{\oplus}^{(1)}, \mathscr{M}_{\oplus}^{(0)}$, and $\mathscr{M}_{\oplus}^{(\lambda)}$ for different $\varkappa$.
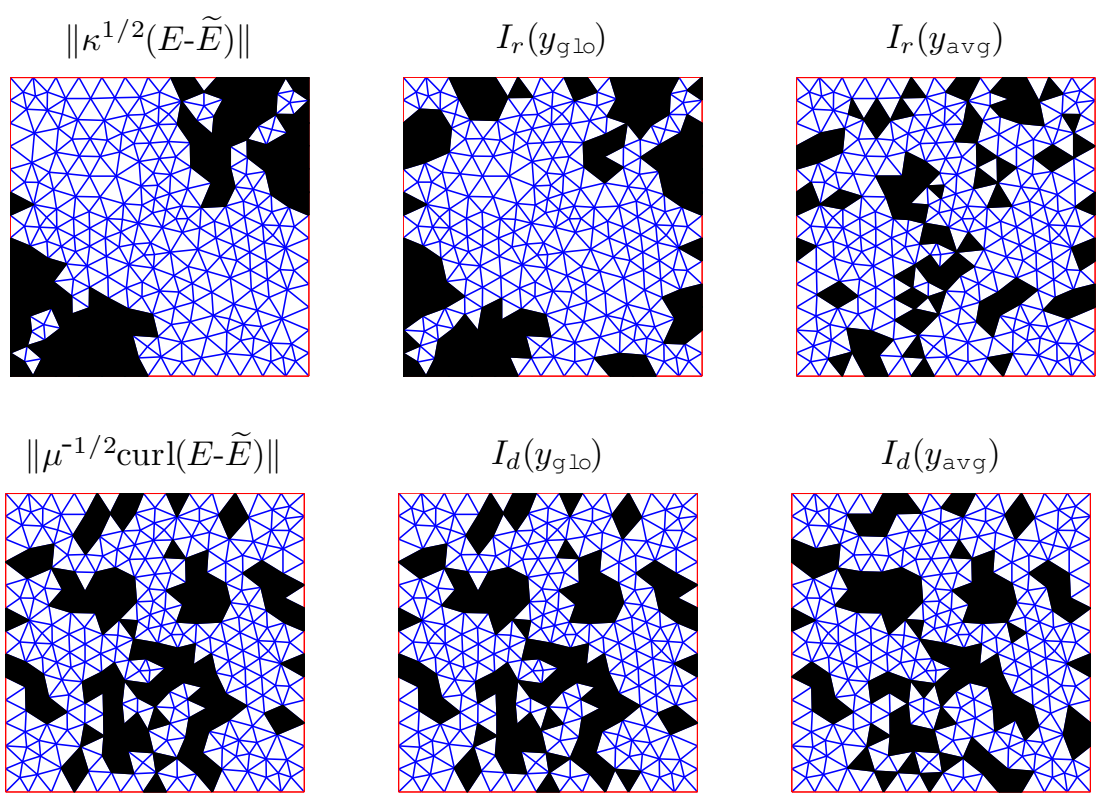

Figure 2. Problem (3.3): Performance of error indicators. 

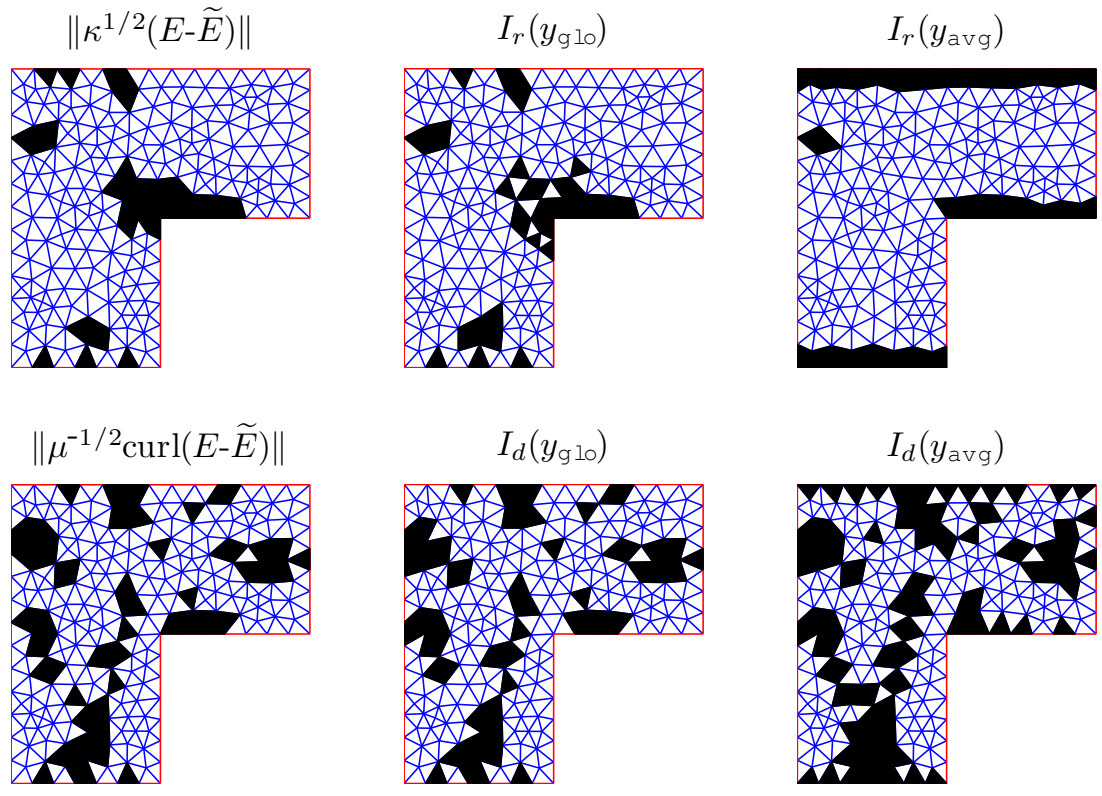

Figure 3. Problem (3.4): Performance of error indicators.

linear $y$ is slow, but using quadratic elements for $y$ we clearly see that the upper bound converges to the exact error. Also, calculating the free parameter in the lower bound $\mathscr{M}_{\ominus}$ in the refined meshes shows that the lower bound is also sharp. From these results we can conclude that one can achieve arbitrary accuracy for the bounds if one is willing to use some time to compute the free parameters in the bounds.

For the second test example we take

$$
\Omega=[0,1]^{2} \backslash\left(\left[\frac{1}{2}, 1\right] \times\left[0, \frac{1}{2}\right]\right), \quad \mu \equiv 1, \quad \varkappa=1, \quad \mathbf{f}=\left(\begin{array}{l}
1 \\
0
\end{array}\right) .
$$

For this problem we do not know the exact solution. A reference solution was calculated in a mesh with 286114 elements. Table 3 gives the efficiency index values for the three upper bounds with some mesh sizes. An approximate solution was computed for each mesh and post-processed so that its divergence becomes square summable. The free parameter $y$ was calculated with linear elements in the same mesh.

Figures 2 and 3 present the error indication results for indicators (3.1) and (3.2). Here, the function $y$ was selected in two different ways: $y_{\text {glo }}$ denotes the function obtained by global minimization of the majorant $\mathscr{M}_{\oplus}^{(1)}$, and $y_{\text {avg }}$ denotes the function obtained by the simple averaging procedure described earlier. The free parameter $y_{\text {glo }}$ was calculated with linear elements in the same mesh in which the approximate solution was calculated. In Figs. 1-3 we have marked with black color all elements with an error greater than the average error. The top rows present the results for 
indicator (3.1) and the bottom rows for indicator (3.2). In each row the first picture shows the exact error distribution that the indicators are supposed to indicate. The second picture shows the result of the indicator with $y_{\text {glo }}$, and the last picture shows the result for the same indicator with $y_{\text {avg. }}$. Generally we observe good performance with $y_{\text {glo }}$. With $y_{\text {avg }}$ the indicators do not perform so well.

\section{References}

1. R. Beck, R. Hiptmair, R. Hoppe and B. Wohlmuth, Residual based a posteriori error estimators for eddy current computation. Math. Model. Numer. Anal. (2000) 34, No. 1, 159-182.

2. D. Braess and J. Schöberl, Equilibrated residual error estimator for Maxwell's equation (to appear).

3. A. Hannukainen, Functional type a posteriori error estimates for Maxwell's equations. Proceedings of ENUMATH 2007 Conference, 2008, pp. 41-48.

4. P. Houston, I. Perugia, and D. Schötzau, An a posteriori error indicator for discontinuous Galerkin discretizations of $H($ curl)-elliptic partial differential equations. J. Numer. Anal. (2007) 27, No. 1, 122-150.

5. S. Nicaise, On Zienkiewicz-Zhu error estimators for Maxwell's equations. Comptes Rendus Mathematique (2005) 340, No. 9, 697-702.

6. P. Monk, A posteriori error indicators for Maxwell's equations. J. Comp. Appl. Math. (1998) 100, 173-190.

7. P. Monk, Finite Element Methods for Maxwell's Equations. Clarendon Press, Oxford, 2003.

8. J. C. Nédélec, Mixed Finite Elements in $R^{3}$. Numeriche Matematik (1980) 35, 315-341.

9. P. Neittaanmäki and S. Repin, Guaranteed error bounds for conforming approximations of a Maxwell type problem (in print).

10. P. Neittaanmäki and S. Repin, Reliable Methods for Computer Simulation. Error Control and a posteriori Estimates. Elsevier, Amsterdam, 2004.

11. S. Repin, A Posteriori Estimates for Partial Differential Equations. Walter de Gruyter, Berlin, 2008.

12. S. Repin, Functional a posteriori estimates for Maxwell's equation. J. Math. Sci. (N. Y.) (2007) 142, No. 1, 1821-1827.

13. J. Saranen, On an inequality of Friedrichs. Math. Scand. (1982) 51, No. 2, 310-322. 